\title{
Handgrip Strength in Individuals with Long- Standing Type 2 Diabetes Mellitus: A preliminary report
}

\author{
Ezema C.I., ${ }^{1}$ Iwelu E.V., ${ }^{2}$ Abaraogu U.O., ${ }^{3}$ Olawale O.A. ${ }^{3}$ \\ ${ }^{1}$ Department of Medical Rehabilitation, University of Nigeria, Enugu Campus, Enugu State \\ ${ }^{2}$ Department of Physiotherapy, University of Nigeria Teaching Hospital, Itiku-Ozall, Enugu State \\ ${ }^{3}$ Department of Physiotherapy, College of Medicine, University of Lagos, Idi-Araba, Lagos
}

Correspondence:

U.O. Abaraogu•E-mail: uabaraogu@yahoo.com

\begin{abstract}
SUMMARY
Adequate muscle strength is required for optimum productivity and low muscle strength is a predictor of physical limitations. Individuals with long-standing type 2 diabetes mellitus have been found to have an increased risk of developing functional disabilities. Handgrip strength is a reliable measurement of the disability index. This study was designed to determine the effect of type 2 diabetes on handgrip strength in adults.

Twenty adult patients with a clinical diagnosis of type 2 diabetes mellitus (10 males, mean age: $52.9 \pm 9.01$ years and 10 females, mean age: $52.6 \pm 5.71$ years) and 20 apparently healthy adults (10 males, mean age: $53.1 \pm 8.94$ years and 10 females, mean age 54.5 yrs \pm 5.56 years) who met the inclusion criteria participated in the study. Handgrip strength was measured with an isometric hand dynamometer and comparisons were made between diabetic and non-diabetic males as well as between diabetic and non-diabetic females. Thev independent t-test were used to analyse the significance difference in hand grip strength between the diabetic and the non- diabetic subjects $(\mathrm{p}=0.05)$.

Results showed significant differences in the mean handgrip strength between the male diabetic and nondiabetic subjects $(p<0.004)$, as well as between the female diabetic and non-diabetic subjects $(p<0.002)$. Long-standing type 2 diabetes mellitus seems to result in a decrease in handgrip strength in both male and female adults. This physical limitation may contribute to low productivity in people with type 2 diabetes mellitus.
\end{abstract}

KEY WORDS: Type 2 diabetes mellitus, handgrip, muscle strength, disability

\section{INTRODUCTION}

Diabetes mellitus is a chronic metabolic condition characterized by persistent hyperglycaemia, with resultant morbidity and mortality (Cohen, 2007). Although there is a paucity of data on the prevalence of diabetes in Africa, available data suggest that diabetes is emerging as a major health problem (Jean et al, 1996). According to the International Diabetes Federation (IDF), the prevalence of type 2 diabetes exceeds 250 million worldwide, with Africa accounting for at least 10 percent of this number, with a minimum of six million sufferers in Nigeria (Chiejina, 2009).

Muscle weakness has been associated with type 2 diabetes, even among subjects with high body mass indices (Balogun et al, 1991; Bohannon, 2001; Clerke and Clerke, 2001). Helmersson et al (2004) attribute this to insulin resistance and hyperglycaemia, which cause a reduction in the number of mitochondria in the muscle cells, a decrease 
in glycogen synthesis and an increase in the amount of circulating systemic inflammatory cytokines, all of which have a detrimental effect on the skeletal muscles. The relationship between muscle contractile functions and force generation on one side and hyperglycaemia had earlier been proposed by Helander et al (2002). Furthermore, Deal (1998) associated the duration of diabetes ( $>6$ years) and poor glycaemic control with even poorer muscle quality and an increased incidence of musculoskeletal conditions like carpal-tunnel syndrome, muscle atrophy and Duputyren's contracture. However, little is known of the trend between long duration type 2 diabetes and handgrip strength in southeast Nigeria. This study was therefore designed to compare the handgrip strength of individuals with long standing type 2 diabetes with the handgrip strength of their apparently healthy age-matched counterparts. This is a preliminary report that precedes a longitudinal cohort study on this population.

\section{METHODOLOGY}

\section{Subjects}

Forty subjects participated in the study, and were divided into two groups (four subgroups, numbering 10 each). Group A consisted of 20 (10 males and 10 females) apparently healthy adult staff of the University of Nigeria Teaching Hospital (UNTH), Ituku Ozalla and the University of Nigeria Enugu Campus (UNEC), all in Enugu State, southeast Nigeria. Group B consisted of 20 (10 males and 10 females) type 2 diabetes mellitus patients attending the diabetic clinic of UNTH, who had been clinically diagnosed as diabetics for at least 6 years before the commencement of this study. Information on the reported age at diagnosis was used to define diabetes duration. Plasma glucose for the control group was measured using an automated glucose oxidase reaction (Vitros 950 analyzer; Johnson \& Johnson, Rochester, NY), to screen out those that were diabetic even without knowing it. Subjects were all right-handed, had no history of upper limb amputation and fell between the ages of 39 to 65 years. None of the participants was involved in an occupation that required manual handling or sports that may have conferred on them the advantage of a better handgrip. There was no difficulty performing basic activities of daily living and no reported use of walking aids. All the diabetic subjects were either on only hypoglycaemic agents, or hypoglycaemic agents and a diabetes diet formula. None of the subjects was on hospital admission at the time of the study. All participants gave written informed consent to participate in the study.

\section{Materials}

1. Saehan hydraulic dynamometer: model SH5001 (Saehan Corporation, Mansan, South Korea) This was used to measure the hand grip strength.

2. Automated glucose oxidase reaction (Vitros 950 Analyzer; Johnson \& Johnson, Rochester, NY) This was used to check the diabetic status of the control group.

3. Weighing scale (Hanson, Ireland) This was used to measure the weight of the participants to the nearest 1.0 kilogramme $(\mathrm{kg})$. It has a range of $0-120 \mathrm{~kg}$.

4. Height Meter (Secca, England) This was used to measure the height of the participants to the nearest $0.1 \mathrm{~cm}$. It has a range of $0-200 \mathrm{~cm}$.

5. Time piece: A calibrated lugar, lug 2150 (made in Germany). This was used for timing the period of contractions and the period between contractions.

\section{Procedure}

Ethical approval for the study was obtained from the UNTH Health Research and Ethics Committee. Written consent was obtained from the participants prior to the commencement of the study. At the beginning of the study, demographic data (i.e., age, sex, weight, and height) were obtained and recorded. Height and body weight were measured on a height metre and a calibrated weighing scale, with the subjects not wearing shoes. The measurement of handgrip strength was done in the waiting room outside the consulting rooms of the diabetic clinic. The American Society of Hand Therapists standardized arm position for handgrip testing was utilized. Each subject was positioned in a straight back chair (about $46 \mathrm{~cm}$ in height, without an arm rest) with both feet flat on the floor. The arm position was demonstrated to the subjects. Each subject was asked to place the right hand on their right thigh and assume a position of adducted and neutrally rotated shoulder, the elbow in approximately 90 degree flexion, with the forearm and wrist in neutral position, and the fingers flexed for the needed maximum contraction. They were instructed to breathe in through the nose and exhale through a pursed lip after a maximum grip effort was made. A demonstration of maximum handgrip strength was given to each subject before they were asked to do it themselves. Each subject was instructed to squeeze the handle of the dynamometer, which was placed vertically in their hands, as hard as possible. The period of the effort did not exceed 5 seconds. A period of 30 seconds rest was given between two trials 
for the dominant (right) hand to be tested and the average of the two trials was taken.

\section{Data analysis}

Data collected were analysed using SPSS (Version 15). The results were presented using descriptive statistics: mean, standard deviation and range. The independent t-test $(p=0.05)$ was used to assess the significance in the difference between handgrip strength among the different groups.

\section{RESULTS}

The demographic characteristics of the subjects are shown in table 1 . The age ranges for the male groups were $39-65$ years for the diabetic group (MDG) and 41 - 64 years for the male non-diabetic group (MNG). The mean ages of subjects in the MDG and the MNG were 52.90 \pm 9.01 years and $54.40 \pm 5.56$ years respectively. The BMI values ranged from $21.98-27.38$ with a mean value of $25.10 \pm$ 1.50 in the MDG and 24.17 - 30.47 with a mean value of $27.97 \pm 2.30$ (table 1). Handgrip strength ranged between $36.5-48.7$ with a mean value of $43.48 \pm 3.39$ for MDG and 43.5 - 55.9 with a mean value of $48.77 \pm 3.86$ for MNG. The independent t-test analysis showed that there was a significant difference between the mean handgrip strength of the male diabetic group and the non-diabetic group $(\mathrm{p}<0.004$, table 2$)$.

Table 1. Demographic and anthropometric characteristics of the subjects $(n=40)$

\begin{tabular}{|c|c|c|c|c|c|c|c|c|}
\hline & \multicolumn{4}{|c|}{ Female $(\mathrm{n}=20)$} & \multicolumn{4}{|c|}{ Male $(\mathrm{n}=20)$} \\
\hline & \multicolumn{2}{|c|}{ Diabetic $(\mathrm{n}=10)$} & \multicolumn{2}{|c|}{ Non-diabetic $(\mathrm{n}=10)$} & \multicolumn{2}{|c|}{ Diabetic $(n=10)$} & \multicolumn{2}{|c|}{ Non-diabetic $(\mathrm{n}=10)$} \\
\hline & Mean $\pm S D$ & Range & Mean \pm SD & Range & Mean \pm SD & Range & Mean \pm SD & Range \\
\hline Age (yrs) & $52.60 \pm 5.71$ & $45-65$ & $54.4 \pm 5.56$ & $46-63$ & $52.9 \pm 9.01$ & $39-65$ & $54.40 \pm 5.56$ & $41-64$ \\
\hline Height $(\mathrm{cm})$ & $164.60 \pm 6.90$ & & $160.5 \pm 4.64$ & & $176.8 \pm 7.92$ & & $169.1 \pm 7.57$ & \\
\hline Weight (kg) & $66 \pm .7 .86$ & $58-87$ & $75.8 \pm 7.39$ & $58-82$ & $78.4 \pm 5.79$ & $69-87$ & $79.9 \pm 7.03$ & $65-89$ \\
\hline $\operatorname{BMI}\left(\mathrm{kg} / \mathrm{m}^{2}\right)$ & $28.37 \pm 3.50$ & & $29.44 \pm 2.96$ & & $25.10 \pm 1.50$ & & $27.97 \pm 2.30$ & \\
\hline
\end{tabular}

Table 2. Statistical analysis of handgrip strength of the subjects

\begin{tabular}{l|l|l|l|l|l|l}
\hline & $\begin{array}{l}\text { Diabetic } \\
\text { Mean } \pm \mathrm{SD}\end{array}$ & $\begin{array}{l}\text { Non-diabetic } \\
\text { Mean } \pm \mathrm{SD}\end{array}$ & $\mathrm{N}$ & $\mathrm{df}$ & Independent t-test & P-value \\
\hline Male & $43.48 \pm 3.39$ & $48.77 \pm 3.86$ & 20 & 18 & -3.256 \\
Female & $27.05 \pm 1.39$ & $31.25 \pm 3.04$ & 20 & 18 & -3.705 & $0.004 *$ \\
\hline
\end{tabular}

The age ranges for the female groups were 45 - 65years with a mean value of $52.60 \pm 5.71$ for the diabetic group (FDG) and 46 - 63 years with a mean value of $54.40 \pm$ 5.56 for the non-diabetic group (FNG) (table 1). The FDG had BMI values ranging from 22.1 - 33.3 with a mean value of $28.37 \pm 3.50$, while the FNG had BMI values falling between $23.8-34.58$ with a mean value of $29.44 \pm$ 2.96 (table 1). The handgrip strength for the female groups ranged from $24.0-29.7$ with a mean value of $27.05 \pm 1.39$ for the FDG and 24.5 - 34.6 with a mean value of $31.25 \pm$ 3.04 for FNG. There was a significant difference in the mean values of handgrip strength of the FDG and FNG ( $p$ $<0.05)$.

\section{DISCUSSION}

The age range of participants in this study, shows that type 2 diabetes mellitus occurs in adults at an earlier age. There was an abnormally high BMI $\left(>25 \mathrm{~kg} / \mathrm{m}^{2}\right)$ among the male and female non-diabetic groups, suggesting a latent risk for development of type 2 diabetes even among the apparently healthy subjects. Our study clearly demonstrated that upper limb muscle quality was consistently lower in adults with a long duration ( $>6$ years) of type 2 diabetes, regardless of sex. This finding plausibly explains the increased risk of upper limb functional limitations in older individuals with long-standing type diabetes. Although we were able to match the control subjects' sex, age and physical activity, 
we could not say whether muscle weakness in type 2 diabetics was due to reduced muscle mass or poor muscle quality because upper limb muscle mass was not assessed.

This study particularly showed that type 2 diabetes may be occurring at an earlier age than previously thought. It is also possible that individuals (especially where routine medical checkup is not a common practice) may have subclinical type 2 diabetes for many years without knowing until hyperglycaemic and neuropathic symptoms manifest. This was the discovery during the screening for the control group. In this instance, age of diagnosis is usually delayed and may pose some difficulty in tracking the effect response trend between duration of diabetes and muscle quality. A backward extrapolation in a cohort study shortly after the clinical diagnosis of type 2 diabetes has strongly suggested that beta-cell dysfunction commences at least 10 years before hyperglycaemia develops (UK Prospective Diabetes Study, 1995; Temelkova, 2002). However, Boyle et al (2001) had earlier found that most of the diabetics in the developed countries are over 60 years.

The mean BMI values of $25.10 \pm 1.50$ in the diabetic males and $27.97 \pm 2.30$ in their non-diabetic counterparts suggest abnormally high BMI values in the non-diabetic males compared to their diabetic counterparts. This could be due to the weight loss associated with long-standing diabetes; or some of the diabetic individuals were on a dietary formula and, therefore, may have had the benefit of weight control. The mean BMI values of $27.97 \pm 2.30$ and $29.44 \pm 2.96$ for the apparently healthy male and female groups could imply a latent risk for the development of type 2 diabetes among the apparently healthy subjects. One would expect that this would translate to a better handgrip in these individuals when compared to their non-diabetic counterparts. However, the reverse, surprisingly, was the case.

Aponectin, an adipocytokine, which plays an important role in carbohydrate and lipid metabolism, and also as a major modulator of insulin action, is characterized by lower circulating levels in the presence of obesity (Chandran et al, 2003). Also, subclinical neuropathic processes involving motor neurons might be another possible underlying mechanism for the poor muscle function in long-standing diabetes (Lesniewski et al, 2003). The mean handgrip strength of the non-diabetic males was higher than the value for their diabetic counterparts, which stood at 43.48 . There was also a higher mean handgrip strength in the female nondiabetics when compared to the value of the diabetic females. These differences in the mean handgrip strength were also found to be significant at $p<0.05$. Insulin resistance may have been responsible for the muscle weakness (Sayer et al, 2005) and, therefore, the decreased grip strength. Rantanen et al (1999) and Leveille et al (2004) have independently reported decreased handgrip strength in individuals with type 2 diabetes mellitus. However, Andersen et al (1997) and Andersen et al (2004) opposed this view, insisting that grip strength is not compromised in long-standing diabetes type 2. These differences in the reports may be due to the lack of baseline record of grip strength in all the studies, thereby making it impossible for the change in grip strength after the onset of diabetes to be determined.

The major limitation of this study was the sample size. Also, cross-sectional studies like this, at best, establish associations between variables but never a cause and effect relationship. Furthermore, no data exists on muscle quality at baseline, which may have an important mediating role in determining future muscle quality of individuals with type 2 diabetes. The study, however, has clinical implications for rehabilitation teams because individuals with longstanding diabetes are at increased risk of developing physical disability. If handgrip assessments are done for a diabetic at the time of diagnosis and routine monitoring incorporated during clinical visits, the development of disability can be detected and preventive modalities like resistive training exercise programme can be instituted to decelerate the rate of deterioration of muscle function before it is too late. Also, the fact that few of our control group subjects were excluded from this study on the basis that they showed evidence of diabetes goes to show that the prevalence of diabetes in our population may have been under-reported.

\section{CONCLUSION}

Type 2 diabetes is associated with poorer upper limb muscle strength and quality. These features may contribute to upper limb functional limitation and physical disability in individuals with long-standing type 2 diabetes. Cohort studies, with a larger sample size, which measure grip strength as well as muscle mass at the point of diagnosis are needed to determine if long-standing type 2 diabetes is associated with a longitudinal decline in upper limb strength and to evaluate the association between loss of muscle mass and muscle quality in people with type 2 diabetes mellitus. 


\section{ACKNOWLEDGEMENT}

The authors immensely appreciate the cooperation and help of the physicians at the diabetic clinic of UNTH.

\section{References}

Anderson, H., P.C. Gadeberg, B. Brock and J. Jakobsen 1997. Muscular atrophy in diabetic neuropathy: A stereological magnetic resonance imaging study. Diabetologia 40: 1062 -106 .

Andersen, H., S. Nielsen, C.E. Mogensen and J. Jakobsen 2004. Muscle strength in type 2 diabetes. Diabetes 53: $1543-1548$.

Balogun, J.A., C.T. Akomolafe and L.O. Amusa 1991. Effect of testing posture and elbow position. Archives of Physical Medicine and Rehabilitation 72: 280-283.

Bohannon, R.W. 2001. Dynamometer measurements of handgrip strength predict multiple outcomes. Percept Motor Skills 93(2): 323-8.

Boyle, J.P., A.A. Honeycutt, K.M. Narayan, T.J. Hoerger, L.S. Geiss, H. Chen and T.J. Thompson 2001. Projection of diabetes burden through 2050: Impact of changing demography and disease prevalence in the US. Diabetes Care 24:19361940.

Chandran, M., S.A. Phillip, T. Ciaraldi and R.R. Henry 2003. Adiponectin: More than just another fat cell hormone? Diabetes Care 26:2442-50.

Chiejina, A. 2009. Diabetes Mellitus: Fast Becoming An Epidemic. Summit on Changing Diabetes in Nigeria. Lagos, Nigeria.

Clerke, A. and J. Clerke.2001. A literature review of the effects of handedness on isometric grip difference of the left and right hands. American Journal of Occupational Therapy 55(2): 20611.

Cohen, Z. 2007. Type 2 diabetes. www.accessscience.com. Accessed on 8/6/2011.
Deal, C. 1998. The Endocrine System: Oxford Textbook of Rheumatology. $2^{\text {nd }}$ edition. London: Oxford University Press.

Helander, I., H. Westerblad and A. Katz 2002. Effects of glucose on contractile function, $\left[\mathrm{Ca}^{+}\right] \mathrm{i}$, and glycogen in isolated mouse skeletal muscle. American Journal of Cell Physiology 282: 1306-1312.

Helmersson, J., B. Vessby, A. Larsson and S. Basu 2004. Association of type 2 diabetes with cyclooxygenase-mediated inflammation and oxidative stress in an elderly population. Circulation 109: 1729 -1734.

Jean, M., F. Claude and B.K. Nagan 1996. Guidelines for the Management of NIDDM in Africa. A Consensus Document. Greece, Novo Nordisk.

Lesniewski, L.A., T.A. Miller and R.B. Armstrong 2003. Mechanisms of force loss in diabetic mouse skeletal muscle. Muscle and Nerve 28: 493 -500.

Leveille, S.G., L.P. Fried, W. McMullen and J.M. Guralnik 2004. Advancing the taxonomy of disability in the older adults. Journal of Gerontology 59:86-93.

Rantanen, T., J.M. Guralnik, D. Foley, K.. Masaki, S. Leveille, J.D. Curb and L. White 1999. Midlife hand grip strength as a predictor of old age disability. JAMA 281: $558-560$.

Sayer, A.A., E.M. Dennison, .H.E. Syddall, H.J. Gilbody, D.I. Phillips and C. Cooper 2005. Type 2 diabetes, muscle strength, and impaired physical function: The tip of the iceberg? Diabetes Care 28:2541-2.

Temelkova, K.T., E. Henkel, C. Koehler, K. Karrei and M. Hanefeld 2002. Subclinical inflammation in newly-detected type 2 diabetes and impaired glucose tolerance. Diabetologia 45: 151.

UK Prospective Diabetes Study Group 1995. UK Prospective Diabetes Study 16. Overview of six years' therapy of type 2 diabetes: A progressive disease. Diabetes 44:1249-48. 